\title{
L'engagement corporel : une alternative au concept polythétique de « sports à risque $»$ en sciences sociales ${ }^{\star}$
}

\author{
Guillaume Routier ${ }^{1}$ et Bastien Soulé ${ }^{2}$ \\ 1 Grupo de Estudios Sociales y Humanistas en Actividad Física y Deporte (ESHAFYD), Facultad de Ciencias de la Actividad \\ Física y del Deporte (INEF), Universidad Politécnica de Madrid (UPM), Madrid, Espagne \\ 2 Centre de Recherche et d'Innovation sur le Sport (CRIS), Université Claude Bernard - Lyon 1, Lyon, France
}

Reçu le 9 septembre 2011 - Accepté le 24 avril 2012

\begin{abstract}
Résumé. Bien que les notions de «sports à risque» et «sports extrêmes » soient couramment utilisées dans la recherche en sciences sociales, il n'existe pas de véritable consensus sur leur signification; les deux termes sont même problématiques. De plus, les pratiquants des activités sportives ainsi désignées rejettent majoritairement ces appellations. Le but de cette contribution est 1) de souligner l'inadaptation de ces notions, 2) de développer en profondeur une définition « alternative », parfois mobilisée par les chercheurs, mais à peine conceptualisée : «l'engagement corporel ». Dans ce cas précis, «l'engagement » peut être compris à la fois comme un comportement et comme une décision. Deux dimensions doivent en effet être distinguées : la première met l'accent sur « l'action », tandis que la seconde se concentre sur «l'être ». Une dimension pragmatique, l'autre identitaire.
\end{abstract}

Mots clés : Conceptualisation, engagement corporel, sports à risque, action, pragmatisme, identité

\begin{abstract}
The corporal engagement: an alternative to the polythetic concept of "risky sports" in social sciences.

Such notions as "risky" and "extreme" sports are commonly used in social science research, although there is no real consensus on their meaning; both terms are problematical. In addition, participants in sports which are labeled as "risky" and/or "extreme" usually reject such characterizations. The purpose of this contribution is 1) to underline the unsuitability of such notions; 2) to develop indepth an "alternative" definition, sometimes mobilized by researchers, but hardly conceptualized: "corporal engagement". In this specific case, "engagement" can be understood both as a behavior and a decision. Two dimensions have to be distinguished: the first one focuses on the "action", while the second one focuses on the "being". That is the reason why it is interesting to conceptualize "corporal engagement" through its pragmatic and identity dimensions.
\end{abstract}

Key words: Conceptualization, corporal engagement, risky sports, action, pragmatism, identity

\section{Introduction}

Depuis les années 1990, de nombreux travaux de sciences sociales soulignent le développement et la radicalisation des expositions sportives au danger (Le Breton, 2002; Stranger, 1999), notamment à travers des activités dites de nature ou de plein air : pratiques ascensionnelles, pa-

\footnotetext{
* Une grande partie de cet article a fait l'objet d'une communication intitulée «Physical engagement : attempt of conceptualization around dangerous sports $»$ lors de la Conférence annuelle de l'Association Internationale de Sociologie du Sport (ISSA) organisée pendant le XVIIe Congrès de l'Association Internationale de Sociologie (ISA) intitulé « Sociology on the move » à Göteborg (Suède) en juillet 2010.
}

rachutisme et $B A S E$ jump, surf, raids aventure, plongée, etc. Que l'on privilégie l'analyse des raisons menant à cette confrontation à l'incertitude, la saisie du sens convoqué par l'expérience du risque, ou encore la genèse des représentations du danger, les termes de «sport à risque » et de «sport extrême » sont fréquemment mis à contribution dans de tels travaux.

Pourtant, le bon sens suffit à se convaincre des ambiguïtés soulevées : doit-on, pour caractériser une activité sportive de risquée, se baser sur la fréquence des accidents, la criticité des traumatismes effectifs ou potentiels, les représentations du grand public, la structure interne de l'activité, son environnement, ou encore le ressenti émotionnel des pratiquants? Par ailleurs, 
un sport extrême est-il extrêmement dangereux, fatigant, difficile, spectaculaire, ou effrayant? Autant d'impasses terminologiques qu'il convient de contourner, sans pour autant confiner au relativisme notionnel. Ainsi, cherchant à adopter une posture sociologique non réductionniste (Berthelot, 2002; 2003), nous préconisons une émancipation vis-à-vis de telles appellations, et proposons une conceptualisation originale : l'engagement corporel.

Cette contribution entend justifier l'usage de ce concept alternatif, ce qui suppose au préalable un développement des raisons ayant conduit à ne pas se satisfaire des définitions des «sports à risque » ou « extrêmes » recensées dans la littérature. Ensuite, notre proposition sera détaillée, et la démonstration de son opérationnalité heuristique entreprise.

\section{Des raisons plurielles de s'émanciper des catégories de sports «à risque » et « extrêmes »}

\subsection{Le surprenant succès scientifique d'une catégorie d'origine médiatique et commerciale : les «sports extrêmes »}

La notion de «sport extrême » s'est imposée avec une rapidité déconcertante au sein du vocabulaire sportif, au point de devenir un néologisme incontournable. Les acceptions de ce terme sont très diverses, car il est étendu à une pléthore d'activités et de spectacles, permettant de créer une apparente similitude entre des pratiques très différentes (Kay \& Laberge, 2003). Les tentatives de définition ne sont guère convaincantes : dilution du sens, redondance par rapport à des catégories préexistantes, relativisme excessif, voire incohérence pure et simple (Soulé, 2008). À tel point que l'on peut clairement mettre en doute la pertinence et l'apport heuristique de cette catégorie d'analyse. Quelle en est l'utilité, quand d'autres notions lui sont à la fois substituables et bénéfiques sur le plan de la clarté? La façon dont ce terme est généralement mis à contribution semble révélatrice d'une concession au langage courant, voire d'un effet de mode (Kusz, cité dans Whearon, 2004) consistant à placer sous la bannière de l'extrême des activités disparates, peu ordinaires, non conventionnelles, acrobatiques, ascétiques et/ou plus ou moins risquées.

Il existe ainsi des termes dont l'emploi est fréquent dans l'intercompréhension quotidienne, et dont l'usage s'impose au sein de la communauté scientifique. Cette simplicité d'emprunt entraîne souvent le contournement des principes énonciatifs de définition (Passeron, 2006); dès lors, les limites conceptuelles de ces objets préconstruits sont fréquemment outrepassées, et l'inconsistance guette. L'éclatement des significations, les ambiguités sémantiques, la faible connotation distinctive et la redondance avec plusieurs autres caractérisations sportives ne permettent ainsi guère l'accession de l'appellation « sport extrême » au rang de concept (Soulé, 2008).

\subsection{Le risque en sociologie : une notion problème au sein d'une discipline confrontée à des difficultés de définition}

Travailler sur le risque engage sur un terrain conceptuel glissant (Jardine \& Hrudey, 1997; Renn, 1998). D'où la nécessité de disposer d'une certaine maîtrise théorique et conceptuelle de cette notion, d'autant que la sociologie est confrontée à sa propre contemporanéité, du fait de son caractère de science historique ${ }^{1}$. Si le choix des mots en sciences sociales est particulièrement délicat, c'est en effet parce que les notions mobilisées ont presque toujours été préalablement utilisées, selon des acceptions fort diverses, y compris dans le langage courant (Bajoit, 2003). Par conséquent, se saisir sociologiquement d'un objet implique une reconstruction qui ne va pas de soi, du fait de la double herméneutique auxquelles les sciences sociales sont sujettes (Giddens, 1987) : elles doivent non seulement construire leur objet, mais également tenir compte des diverses interprétations (de caractère spontané ou savant) préexistant à l'observation (Duchastel \& Laberge, 1999).

\subsection{Les sous-entendus de la notion de « sport à risque »}

Un bref rappel des fondements sur lesquels se construisent les significations implicites de l'expression « sport à risque » suffit à se convaincre de la pertinence du constat dressé dans ce sous-titre : fréquente assimilation des termes danger et risque, gommant les processus de subjectivation conduisant du premier au second ${ }^{2}$; recours à l'énumération d'activités, conduisant à un amalgame peu cohérent de sensations, d'aventure, de violence, de vitesse, d'acrobaties, de jeu avec la gravité, d'endurance, de courage et d'environnements hostiles; désignation des sports les plus risqués au regard de la magnitude des éventuels écueils corporels, davantage qu'en référence à leur probabilité d'occurrence; association tacite entre « sports à risque » et registre de la prise de risque volontaire (Soulé \& Corneloup, 2007b). Ainsi, à l'évocation de l'appellation « sport à risque », vient spontanément à l'esprit un type bien particulier d'engagement : la pratique d'activités de « loisir sportif », hors cadre institutionnel et en plein air, dans un environnement propice aux accidents, théâtre de prises de risque pouvant entrainer des blessures graves ou la mort. Plusieurs chercheurs en sciences sociales s'en

\footnotetext{
1 Au sens de Passeron (2006).

2 Selon Pardo (2002), cette distinction est habituellement faite dans d'autres champs, mais de nombreux chercheurs en sociologie du sport prenant comme objet les sports à risque ne lisent pas d'autre littérature que celle ayant directement trait à ce champ d'investigation.
} 
Encadré 1. Synthèse terminologique.

Pour les sociologues privilégiant une approche structurelle, à l'image de Bouet (1968), Rotillon (2002), Penin (2004) ou encore Martha et Griffet (2006), il est souhaitable de porter prioritairement son regard sur la gravité des écueils corporels induits par certaines activités sportives. Dès lors, la « possibilité de trouver la mort est un critère de hiérarchisation des sports, préalable nécessaire à tout questionnement sur le sens de l'engagement dans l'action » (Martha \& Griffet, 2006, p. 636). La perspective structurelle peut aussi être envisagée à partir des travaux de Parlebas (1981), pour qui la structure ${ }^{\mathrm{a}}$ des sports prédétermine le niveau de risque pour ses adeptes. Collard (1998) va le plus loin, estimant que, pour que l'on puisse parler de sport à risque, il faut que celui-ci présente des processus stochastiques (l'aléa de la situation) et des enjeux (la gravité des écueils éventuels).

Des approches subjectivistes distinguent risques objectifs, dépendant des caractéristiques intrinsèques de la situation, et risques subjectifs, renvoyant au domaine psychoaffectif du sujet. Dès lors, on considère que seuls les acteurs sont en mesure de déceler les principes définitionnels de leurs activités (De Léséleuc \& Raufast, 2004). L'expérience du danger devient le principe définitionnel des activités sportives à risque, certaines étant pratiquées pour le « frisson » procuré (Stranger, 1999), les émotions ou le vertige (Héas, Lebreton, Frezza, Bodin \& Robène, 2007, p. 97).

D'un point de vue culturaliste, toutes les situations porteuses de dangers n'accèdent pas au statut de risque (Gilbert, 2003). Ainsi, si d'une personne à l'autre la perception des risques peut varier, il en est de même d'un groupe à l'autre et d'une société à une autre. «La définition des risques est aujourd'hui essentiellement politique » (Kasperson, cité par Leiss \& Chociolko, 1994, p. 3), car lorsqu'une situation est définie comme risquée, il devient nécessaire pour les populations et gouvernements de s'en occuper et de les résorber. En ce sens les risques sont socialement construits et par conséquent, parmi la multitude de risques auxquels nous faisons face en permanence, seulement certains attirent notre attention (Douglas, 1992).

Enfin, Soulé et Corneloup (2007b) font référence aux définitions systémiques des « sports à risque ». Si ces dernières n'appartiennent pas directement au champ des sciences humaines et sociales, mais aux sciences du danger (autrement appelées cindyniques), elles n'en sont pas moins intéressantes. Kaplan et Garrick (1981) distinguent ainsi à propos du risque ce qui peut arriver (scénario), les chances pour que cela se produise (probabilité) et l'ampleur des dommages éventuels (conséquences). Dans une optique systémique, le danger est alors envisagé comme une conjonction de facteurs se succédant dans le temps. L'analyse des risques sportifs vise à cerner ce faisceau de facteurs et leur enchevêtrement complexe aux différentes étapes du processus de danger.

a Entendue comme la manière dont les traits de logique interne de ce sport (rapport à l'environnement, interaction entre les joueurs, système de réussite, instrument technique utilisé, temporalité, etc.) sont agencés entre eux (Collard, 1998).

contentent du reste, et recourent à cette catégorie sans se parer des précautions terminologiques qu'implique pourtant, a priori, le recours à un terme usuel.

\subsection{Les sports à risques : des définitions variées}

Un certain nombre de formalisations académiques ont néanmoins tenté de clarifier ce qu'était un « sport à risque ». Cinq perspectives conceptuelles (critique, structurelle, culturaliste, systémique et subjectiviste), présentées dans l'encadré 1 et revendiquant plus ou moins fortement leur inscription dans un courant sociologique du sport, peuvent être différenciées (Soulé \& Corneloup, 2007a).

Ainsi, les chercheurs n'abordent les «sports à risque » ni sous le même angle, ni en référence aux mêmes théories et programmes. Du coup, au sein de ce paysage terminologique, les similitudes et complémentarités sont aussi nombreuses que les contradictions et incompatibilités. Cette diversité atteste du caractère polythétique (Needham, 1975) de la notion de « sport à risque », qu'on ne peut dès lors espérer définir que de manière relative et arbitraire.

\subsection{L'illusion d'une définition générique du risque}

Cet état de fait génère néanmoins un malaise chez les partisans d'une épuration terminologique (Boudon, 1990), prompts à considérer cette pluralité comme un problème, et appelant à des tentatives de formalisation unificatrice. Cet a priori selon lequel il est possible d'aboutir à une définition claire et partagée est illustré par l'appel normatif de Collard $(1997 ; 1998)$ à davantage de rigueur conceptuelle, assorti d'une proposition de conceptualisation « objective» de ce qu'il convient d'entendre, stricto sensu, par «sport à risque ».

Prétendre parvenir à une définition générique contraint toutefois à opérer des rapprochements entre des situations relevant de dynamiques extrêmement différentes (Duchastel \& Laberge, 1999) : quel est le degré de similitude entre les modalités d'exposition au danger du rugbyman professionnel, du pratiquant ponctuel du saut à l'élastique et du nageur en piscine publique? Une description réaliste du champ conceptuel consisterait plus humblement à reconnaître sa perpétuelle mobilité (Passeron, 2006). Dans cette perspective, la diversité rencontrée ne constitue pas un problème, devenant la simple preuve d'une inscription disciplinaire et théorique, en 
même temps que le reflet des préoccupations particulières du chercheur (Kaplan, 1997).

\subsection{Au-delà de la catégorie restrictive de « sports à risque »}

Chaque pratique sportive génère des dangers se traduisant par une accidentologie et une traumatologie spécifiques. L'utilité même d'une définition des « sports à risque » se voit ainsi remise en question : sans pour autant considérer toutes les activités sportives comme identiquement dangereuses, qualifier certains sports de risqués conduit implicitement et abusivement à en désigner d'autres ne l'étant pas (Monod \& Kahn, 2005).

Dès les années 1960, la mise en évidence de déterminants structurels désignant des pratiques sportives particulièrement risquées a néanmoins été entreprise (Bouet, 1968), puis affinée (Collard, 1998; Rotillon, 2002 ; Penin, 2004). La présence d'incertitude, d'enjeux corporels ou la possibilité de décès constituent alors des pivots définitionnels. Toutefois, s'engager dans cette voie ne permet guère d'échapper au piège de la normativité. En excluant le sens investi par les sujets dans leur pratique, tout comme les perceptions et représentations individuelles, on s'en tient à caractériser certaines activités de dangereuses. Pourtant, ce qui compte pour quiconque entend mobiliser la notion de risque en sciences sociales, ce ne sont pas tant les caractéristiques intrinsèques de la situation (telles qu'elles sont « objectivées » par l'analyste) que le regard porté sur elles et la manière de les appréhender.

Une autre tentation consiste à «partir des faits », en s'appuyant sur l'accidentologie pour désigner des pratiques particulièrement risquées. Dans cette perspective, le principal obstacle réside dans les difficultés méthodologiques qui fragilisent la constitution d'un tableau d'ensemble satisfaisant. Par ailleurs, on peut là encore objecter que quoique disent les chiffres, c'est bel et bien le sens associé à ces pratiques qui préside à la qualification «à risque» de certaines pratiques. Toutes les situations porteuses de dangers n'accèdent pas au statut de risque; inversement, des activités relativement sûres acquièrent le statut de pratiques risquées.

$\mathrm{Au}$-delà de cette construction sociale, plusieurs écrits inscrits dans les courants phénoménologique ou postmoderne affirment que toute pratique sportive peut être considérée comme risquée aux yeux d'un individu particulier, du fait de l'influence de l'environnement culturel, du travail cognitif et du ressenti qui participent de la reconstruction du risque. Ainsi, il ne peut y avoir de risque sans sujet (individuel ou collectif) pour produire les représentations correspondantes (Coanus \& Pérouse, 1999). Tout l'enjeu pour le chercheur consiste dès lors à comprendre la manière dont de multiples messages (médiatiques, publicitaires, judiciaires, politiques, etc.) sont interprétés par des acteurs qui leur impriment leurs propres convictions, valeurs, connaissances et expériences ${ }^{3}$. C'est de cette « alchimie » que naît la catégorisation « à risque » de certaines modalités de pratique sportive.

Ces positions contrastées ne sont pas sans rappeler la dualité émic/étic en anthropologie (Olivier de Sardan, 1998) : le risque est assimilable à l'émic, centré sur des significations culturelles et lié au point de vue des acteurs; le danger est comparable à l'étic, reposant sur des données dites «objectives » (observations externes indépendantes des significations portées par les acteurs). Il n'est évidemment pas question d'opposer risque et danger, mais de souligner leur imbrication, tant ils constituent pour le sociologue les deux faces d'un seul et même phénomène.

Enfin, la notion de «sport à risque » est fragile du fait du rejet qu'elle suscite chez de nombreux pratiquants (parachutistes, $B A S E$ jumpers, freeriders, alpinistes, etc.). Ils refusent quasi unanimement cette appellation, l'assimilant à un dessaisissement irresponsable de leur sort, alors qu'ils sont au contraire désireux d'afficher la cohérence de leur ligne de conduite et la rationalité de leurs choix (Martha \& Griffet, 2006; Penin, 2004). Privilégier le concept d'engagement corporel permet dès lors d'aborder ces conduites «non comme une manifestation pathologique ou transgressive, mais plutôt comme une réalité ordinaire $»$ (Raveneau, 2006b, p. 583).

\subsection{Prendre ses distances avec les inscriptions programmatiques marquées}

Des tentatives d'objectivation structurelle aux perspectives relativistes et phénoménologiques, en passant par les approches culturaliste, représentationnelle ou systémique, l'existant est varié et d'une indéniable richesse. Au regard de la teneur pluraliste et non réductionniste du travail entrepris sur l'engagement corporel (Routier, 2011), il s'avèrerait incohérent d'adhérer simplement à l'une ou l'autre de ces définitions. L'élaboration d'une appellation alternative, suffisamment ouverte, sans pour autant se révéler poreuse ou s'apparenter à une forme molle de syncrétisme, s'impose donc logiquement ${ }^{4}$.

\section{Le concept $«$ alternatif $»$ d'engagement corporel}

Davantage qu'un ensemble d'activités sportives, l'engagement corporel désigne un type d'exposition au danger,

\footnotetext{
${ }^{3}$ On rejoint ici le point de vue de Duclos (2005, p. 4) : «Le risque, c'est un style d'être au monde, un filtre herméneutique des actes, et certainement pas un attribut des choses qui attendraient, passives, leur agent. »

4 Le concept d'engagement corporel n'ambitionne pas de se substituer à celui de «sport à risque », Cependant, il n'entend pas s'appuyer outre mesure sur l'une ou l'autre des définitions recensées à son propos.
} 
conscient et assumé, dont sont porteuses certaines modalités de pratique. Insister de la sorte sur cette acceptation des dangers ne doit pas conduire à penser qu'elle est envisagée comme la clé de voûte de l'investissement sportif ; elle n'en constitue qu'une facette, dont il s'agit ici de caractériser la teneur plutôt que les motifs. L'engagement pouvant être entendu au double sens de « conduite » et d'« acte » inscrit dans une forme d' « être au monde », il renvoie au « faire » mais aussi à l'« être ». Car l'épaisseur de la conduite s'inscrit dans le sens que l'individu donne à son acte. Par conséquent, il semble opportun de fonder le concept d'engagement sur ses dimensions pragmatique et identitaire.

\subsection{La dimension pragmatique de l'engagement corporel}

La notion d'engagement est fortement liée aux termes « action » et « acte »; comprendre l'engagement nécessite donc de s'attarder sur ce que « s'engager » signifie à travers ces mots. Sous cet angle, il s'agit bien d'un verbe d'action, d'une manière d'agir pouvant être appréhendée à la fois comme acte et comme conduite sociale (Ladrière, 1997).

\subsubsection{Implication et mise en gage corporelle : une acceptation des dangers}

Étymologiquement, le concept d'engagement peut être défini comme «l'action de mettre en gage quelque chose $»^{5}$. Il n'est donc pas gratuit, car il coûte d'une façon ou d'une autre. Ainsi que le soulignent de nombreux pratiquants, ce qui différencie bien souvent leurs activités des traditional sports (Coakley, 2004; Young \& Atkinson, 2008), c'est qu'eux n'ont pas, ou si peu, le droit à l'erreur :

«En fait le problème de ces sports c'est qu'en fait il y a un cadre. Et le cadre il est relativement rigide [...] et dès qu'on en sort, contrairement à d'autres activités [...] on va se faire sanctionner $»$ (Bernard, deltaplaniste).

Envisagé ici sous l'angle moteur et corporel, l'engagement relève d'une décision par laquelle les pratiquants acceptent de mettre en gage leur vie ou leur intégrité physique. De ce point de vue les pratiquants « engagés » se situent davantage sur le pôle de l'acceptation des dangers (auxquels ils se confrontent en toute connaissance de cause $^{6}$ ), que sur celui de la « prise de risque ». En tout état de cause, il ne s'agit pas d'une exposition au danger « par

5 Définition du Centre National des Ressources Textuelles et Lexicales (CNRTL) disponible à sur http://www.cnrtl.fr/ definition/engagement (consulté le 18 mars 2011).

${ }^{6}$ Ce que la formule de Boutroy résume bien : « le risque apparaît comme un attrait repoussant, figure oxymore à la fois valorisée et déniée, acceptée et combattue $»(2006$, p. 599). myopie » ou par inconscience des risques : la rationalité, la responsabilité et la prudence sont mises en avant :

«Voir quelqu'un se tuer, ça m'est déjà arrivé, plusieurs fois malheureusement. Et c'est toujours triste de perdre un camarade, mais ce n'est pas ce qui ...ça te rappelle surtout que tu ne joues pas avec n'importe quoi et ça t'oblige à rester hypervigilant» (Marc, $B A S E$-jumper).

L'engagement nécessite donc d'assumer pleinement la situation qui se joue, dans laquelle on « prend ses responsabilités ». Une telle conduite présuppose tout d'abord que l'individu soit impliqué dans la situation, qu'il s'engage activement dans celle-ci. L'engagement corporel se distingue donc assez nettement des prises de risque spontanées et irréfléchies souvent attribuées à la jeunesse. De même, en aucun cas l'absence de raison, généralement associée à ces conduites, ne peut être assimilée à l'engagement corporel. Celui-ci implique l'idée de réflexivité, qui elle-même implique que les résultats de l'expérience soient sans cesse intégrés à sa construction (Bourdin, 2003). Qui plus est, le danger (non encouru pour lui-même) ne constitue pas une fin en soi et semble plutôt constituer un moyen pour obtenir un bénéfice d'ordre quelconque. L'engagé place alors ses motivations sous la discipline de son projet, contrairement à celui qui s'emporte et voit sa force vitale prendre le dessus sur sa capacité à se contrôler et se projeter.

\subsubsection{Seuil d'engagement et degré d'acceptation des dangers}

Des formes plurielles d'exposition peuvent être observées ; toutes ne peuvent cependant être qualifiées d'engagées. En effet, l'engagement corporel suppose l'autonomie des pratiquants, davantage qu'il ne s'appuie sur une prise en charge sécuritaire extérieure (technologique, pédagogique et/ou logistique). On retrouve notamment (mais pas exclusivement) cette forme d'engagement corporel dans certaines modalités de sports de nature telles que l'alpinisme, le $B A S E-j u m p^{7}$, la plongée en apnée en grande profondeur, etc.

Néanmoins, dans bien des cas, la part belle est faite à la maîtrise sécuritaire structurelle, indépendante du pratiquant et ce y compris dans les sports de nature. En cela, le danger comme sa gestion sont avant tout exogènes, et la prise en charge sécuritaire prend le dessus sur toute autre considération. C'est le cas des activités relevant, selon Poizat (2000), du «paradoxe de la liberté réglementée » se traduisant par une fréquentation accrue de milieux naturels aseptisés à grand renfort de règles, d'équipement et de logistique. Dans ces cas de figure, l'erreur ou la défaillance du pratiquant ne porte

\footnotetext{
7 Acronyme signifiant Building (sauts de tours et autres monuments), Antenna (sauts d'antennes), Span bridge (sauts de ponts) et Earth (sauts de falaise, autrement appelé paralpinisme).
} 
que très rarement à conséquence et relève finalement plus d'un « sensationnel assuré » (Seigneur, 2004) dans le cadre d'une «aventure organisée » (Barthélemy, 2002). Il en est de même pour les activités à propos desquelles la maîtrise de la gestion sécuritaire est confiée à un professionnel censé assurer une sécurité parfaite (baptême de parachutisme, descente en canyonisme avec moniteur, vol en parapente biplace, etc.). Parler d'engagement corporel semble dès lors inadéquat puisque l'exposition est totalement euphémisée. Enfin, il est d'autres activités ne s'inscrivant pas dans le registre de l'engagement corporel, tel que nous entendons le conceptualiser : les activités pour lesquelles la gestion et la maîtrise sécuritaire sont en partie exogènes, à l'exemple d'une course en montagne avec guide, d'une escalade en falaise aménagée, voire d'une via ferrata (Boutroy, 2002). En effet, dans le cadre de ces activités, la sécurité semi-active reste sous couvert d'un dispositif technique/technologique et de la responsabilité d'un encadrant, palliant le manque de compétences et de culture spécifiques de l'individu. Sur ces différents aspects, certaines options éloignent d'un engagement corporel à proprement parler, car le pratiquant n'est pas entièrement autonome.

Ainsi, l'engagement corporel implique une autonomie qui est du reste revendiquée par les pratiquants. Ces derniers privilégient une autonomie décisionnelle et motrice totale dans leur pratique, ce qui implique un vécu important pour bénéficier d'une maîtrise technique et d'un bagage sécuritaire suffisants. Dès lors, dans cette optique, le matériel technique ${ }^{8}$ et la préparation logistique servent moins à éradiquer le danger de l'activité qu'à éviter et atténuer les impacts d'un événement non souhaité (ENS) : mise en place d'une sécurité en kayak de rivière, usage d'un parachute de secours en cas de problème en parachutisme, etc. Dit autrement, le dispositif est moins tourné vers le gommage systématique de l'ENS que vers son évitement et l'atténuation de ses effets, étant entendu que celui-ci ne saurait être totalement évacué du champ des possibles ${ }^{9}$. Si le contrôle exercé sur ces situations motrices n'est jamais total, ce n'est pas pour autant pour s'en remettre à des forces décisionnelles extérieures; il s'agit au contraire de « laisser au sportif la possibilité d'être acteur de son dépassement » (Boutroy, 2006, p. 598).

8 Boutroy (2006) souligne une course à l'équipement technologique censé protéger les himalayistes, très préoccupés par leur sécurité : Gore-Tex, combinaisons d'altitude, chaussures thermiques, masques Néoprène...

9 En haute et très haute montagne, il s'agit au moyen de cordes, baudriers, piolets, pieux à neige, broches à glace et autres pitons, dont l'usage nécessite des gestes adaptés, non pas d'éviter, mais «d'assurer » les chutes (Boutroy, 2006). En kayak de haute rivière, casque, gilet de sauvetage, vêtements rembourrés et corde de sécurité s'imposent de même (Marsac, 2006). Certains de ces objets permettent d'assister, le cas échéant, d'autres pratiquants en difficulté.
Étant donné que l'ENS ne peut être totalement évacué, l'engagement relève donc bien d'une acceptation d'un danger dont le pratiquant cherche à limiter l'impact potentiel par l'acquisition de savoir-faire mentaux et corporels particuliers, l'expérience étant à la fois sensorielle, technique et langagière (Raveneau, 2006b). Il y a bien mise en péril consciente et assumée de l'équilibre sécuritaire. Le « jeu aux limites » (Griffet, 1991) prend dès lors un visage fort différent de ce que l'on constate dans les modalités d'exposition décrites supra : il convient de dépasser ses appréhensions, de vaincre son éventuelle peur, de maîtriser ses émotions, d'avoir des réactions adéquates, de déceler les signes précurseurs de scénarios de danger, etc. Dès lors, l'autonomie et la responsabilité étant recherchées (voire revendiquées), le renoncement fait partie des possibles :

«Tu sais, tu repères un truc d'en bas et tout, ça te paraît nickel, alors tu montes. Tu montes là-haut pendant plusieurs heures à pied. T'arrives et en fait il y a une énorme gonfle et tu te dis ça pue, ça pue. Bah tu fais demi-tour quoi. Je pense qu'il faut rester humble avec la montagne et il faut être capable de renoncer justement $»$ (David, Freerider).

« [Il s'agit] de maintenir un savant équilibre entre la peur, les appréhensions et le plaisir [...] il faut savoir utiliser les deux jauges. Et si la jauge de la peur elle est bien au-delà de la jauge du plaisir, on arrête, on continue pas» (Yves, BASE-jumper).

Notons enfin que certaines formes d'engagement corporel rendent la maîtrise sécuritaire passive totalement impossible : course au large ou en très haute montagne et en solitaire, plongée et apnée libre en grande profondeur, saut en BASE-jump, etc. Dans pareilles circonstances, tout repose sur la vérification en amont du matériel, le degré d'exposition accepté ainsi que sur la stratégie et la maîtrise technique développées par le pratiquant.

«Une survie, on y est préparé [...] on est habitué à ce genre de situation, à ces périodes de stress. On est habitué à gérer, à s'équiper de combinaisons de survie, à utiliser les téléphones Meridian, à lancer des fusées, etc. Vous entrez dans une procédure, quelque chose qui est appris» (Pascal, navigateur).

«De toute façon, même au début, si tu inities quelqu'un tu vas le laisser plier lui-même sa voile. Tu peux lui donner des conseils en fonction de l'exit sur lequel t'es, mais perso je ne prendrais pas la responsabilité de lui plier sa voile. T'imagines si il se fracasse et que c'est toi qui avait mal plié... c'est un truc que tu dois faire seul. En même temps c'est un bon moyen de comprendre qu'il faut faire les choses sérieusement. T'es vachement plus concentré quand tu plies toi-même ce qui te relie à la vie. Tu prends vite tes responsabilités, je te le dis moi!» (Hervé, BASEjumper).

Il convient tout de même de préciser que cette forme exacerbée d'acceptation des dangers n'est pas la seule que 
nous qualifions d'engagement corporel, bien que certains alpinistes, par exemple, opposent de façon binaire exposition (renvoyant à une situation dangereuse en soi, comme le fait de remonter un couloir avalancheux) et engagement (caractérisant une situation limitant ou excluant toute possibilité de retraite ou de secours) (Boutroy, 2006) ${ }^{10}$.

\subsection{La dimension identitaire de l'engagement corporel}

Si l'acceptation des dangers n'est plus à considérer comme une fin en soi, elle ne constitue pas non plus un simple « moyen de se faire valoir ou d'éprouver du plaisir, mais la conséquence d'un cheminement » (Martha \& Griffet, 2006, p. 635) qui imprime un sens particulier à l'acte d'engagement. En effet, ce dernier renvoie à l'audace qui consiste non seulement à sortir des sentiers battus, à se mettre physiquement en jeu, mais aussi à se confronter aux autres, dans un contexte où l'erreur ou l'indécision porte tout particulièrement à conséquence. Á travers l'engagement, on ose se placer et se montrer en situation délicate, sachant que cette dernière démultiplie, d'une part, les enjeux identitaires et interactionnels, et permet, d'autre part, l'expression de soi et de ses affects, moins canalisée qu'à l'accoutumée. Tout engagement est donc une révélation de soi, pour soi-même mais aussi devant l'autre car « la prise de risque revêt aussi une indéniable dimension psychologique dont les enjeux sont l'estime de soi, la réputation personnelle, la virilité, le courage, etc. » (Raveneau 2006a, p. 620). Il peut s'agir de conforter son image propre (ce qu'on pense de soi) et son image sociale (celle que les autres ont de soi). La stratégie est dans ce cas claire : acquérir ou maintenir un statut, la prise de risque étant un facteur de popularité, de prestige, de compétitivité, etc.

S'il porte à conséquence sur le plan identitaire, c'est aussi parce que l'engagement corporel implique son invention et sa création : il est davantage personnalisé et individualisé que collectif, codifié et institutionnalisé. Il constitue ainsi l'expression de sa liberté, à travers cette envie d'agir, mais aussi une reprise de l'initiative sur un terrain atypique. On devient soi-même dans la continuation de ses choix et de ses engagements, on y construit une figure de soi.

\footnotetext{
10 Martha et Griffet (2006) nomment sports létaux ceux qui n'offrent aucune possibilité de réchappe, secours ou assistance. Le sport létal se définit donc par le tout ou rien (Martha \& Griffet, 2006). Ainsi, en BASE jump, l'éventualité d'une mort liée au hasard est écartée : « le joueur ne se fie qu'à lui-même, à la juste appréciation de ses potentialités et du monde qui l'entoure » (Martha \& Griffet, 2006, p. 637) ; « pour les BASE jumpers, il est inconcevable d'effectuer une tentative qui ne puisse trouver, à coup sûr, une issue favorable » (Martha \& Griffet, 2006, p. 638). Ces adeptes rejettent le hasard, cherchant à «atteindre ce sentiment de maîtrise presque totale sans lequel le renoncement au saut s'impose » (Martha \& Griffet, 2006, p. 641).
}

\subsubsection{L'acceptation du danger : une éthique de la responsabilité}

La présence de dangers identifiés et assumés impose $a m i$ nima une double forme de réflexivité; l'une immédiate et pragmatique, nécessitant une réponse rapide dans l'action afin de répondre à une situation éventuellement incertaine; l'autre plus latente, imposant de faire des choix de valeur tels que Weber (1995) a pu les décrire. « L'éthique du risque est une éthique de la responsabilité qui impose à chacun, individuellement et collectivement, de se prononcer au cas par cas sur la valeur des valeurs » (Ewald \& Kessler, 2000, p. 67). Etre responsable (mais aussi reconnu responsable) de ses actes peut alors revêtir une teneur identitaire forte que l'engagement corporel est à même de renforcer. Le risque n'est en cela plus une fin en soi mais bien un moyen d'obtenir des gains d'ordre divers et plus particulièrement identitaires. Du reste, cette volonté individuelle de maîtrise est présente dans la culture contemporaine du risque (Giddens, 1991) et plus largement médiatisée dans le marketing sportif réalisé autour de ces activités corporellement engagées.

La confrontation au danger a donc pour corollaire de rendre certaines décisions particulièrement cruciales, in situ comme au stade de la préparation : mise en œuvre de techniques motrices adaptées; déploiement de dispositifs technologiques adéquats pour éviter l'activation de scénarios de danger, contrarier la poursuite de séquences accidentelles initialisées et/ou minimiser les impacts; renoncement à l'activité projetée, etc. Des connaissances et compétences spécifiques s'imposent dès lors pour anticiper les incertitudes, déceler les signes précurseurs du danger, conserver un contrôle sur le cours des événements et éviter que les situations provoquées ne basculent dans une forme d'instabilité critique. Combinée au contrôle de ses émotions, leur acquisition permet, dans une certaine mesure, de faire face à la mise en suspens de son intégrité corporelle.

Il peut aussi s'agir de s'engager à analyser et gérer collectivement les aléas, « pièges » et difficultés d'une sortie, ce qui implique de se plier à une organisation (Marsac, 2006) et génère une organisation sociale, ainsi qu'une expérience fraternelle particulières (Boutroy, 2006); de s'engager à revenir, pour les autres (ses proches, sa famille) ; ou encore de mettre un point d'honneur à « ramener le bateau » (Liardet \& Raveneau, 2006). À l'image des adeptes de descente de haute rivière en kayak observés par Marsac (2006), d'autres pratiquants évoquent une forme d'engagement à se comporter de la manière prévue après concertation et «lecture » collective de la situation, de façon à assurer sa propre sécurité tout comme celle des autres, éminemment interdépendantes.

«Tous les ans ça arrive, tous les ans y'en a qui se tuent comme ça. Pour $X$ raisons ils se cassent les jambes [...] et ils sont là, y'a personne et puis ils meurent de souffrance ou de froid selon la saison. 
Non, c'est une erreur, c'est une erreur de sauter seul $\gg$ (Marc, BASE-jumper).

«L'avantage d'être en groupe c'est aussi que l'analyse de la situation elle est collective. Donc la prise de décision, même si au fond ça reste toi qui décides, elle est un peu collective. Les autres gardent un œil sur toi et tu gardes un oeil sur eux. Tu peux par exemple faire une lecture collective du couloir que tu vas faire et définir, ensemble, le chemin adéquat. Tu te dis tiens voilà on va passer à droite de tel rocher, à gauche de ci de ça, telle barre on la saute ou on la contourne et tout $»$ (Sylvain, freerider.)

Ainsi, le respect des décisions prises en amont, assimilable à un certain conformisme destiné à «éloigner le danger », peut être érigé au rang de règle sociale, alors que les initiatives individuelles sont à l'inverse bridées. En plongée, on observe de même une appréhension et une gestion collectives des risques. Érigée au rang d'une quasi-éthique de la responsabilité, cette gestion fait de bien des formes d'engagement un monde socialement organisé (Raveneau, 2006b). Dès lors, on constate que cette construction sociale de la responsabilité prend une place importante à la fois dans les discours de pratiquants, mais aussi dans leurs actes. Car, si elle permet d'assurer un haut degré de sécurité, elle favorise aussi et surtout l'expression d'une identité engagée conforme à une identité responsable assignée (Bajoit, 2003) socialement et médiatiquement. En valorisant cela les pratiquants s'assurent ainsi une reconnaissance solide sur laquelle ils construisent leurs images sociales.

\subsubsection{Engagement et unité existentielle}

S'ils accèdent à la reconnaissance en communiquant leur responsabilité, c'est avant tout dans l'action, dans l'engagement pragmatique, que les pratiquants tentent d'unifier et de trouver un équilibre satisfaisant entre leurs différentes identités possibles (désirées, assignées et engagées) (Bajoit, 2003). Dès lors, ce type de structure appelle des possibilités existentielles diverses.

La spécificité de l'engagement vient donc aussi de ce qu'il fait advenir quelqu'un, une personne qui ne lui préexiste pas, tant les pratiquants ne se considèrent plus les mêmes avant et après.

«C'est assez bizarre comme sensation en fait. D'un côté c'est parfois vachement violent, et en même temps y'a un côté hyper profond » (Michaël, alpiniste).

«Plus tu vas profond plus la bulle elle se referme et puis en même temps tu... plus tu te refermes, plus t'as des sensations qui arrivent quoi. Je crois que c'est Mayol qui disait «tu vois les plongeurs bouteilles ils plongent pour regarder autour d'eux, et les apnéistes ils plongent pour regarder à l'intérieur d'eux» [...]
T'es à l'écoute de ton corps, t'es à l'écoute des sensations [...] savoir s'écouter c'est aussi apprendre à se connaître et savoir grandir $»$ (Patrice, apnéiste).

En cela, le risque constitue une ressource identitaire car « la prudence, louable et souvent invoquée, n'est pourtant jamais vraiment valorisée. La prise de risque en revanche est plus souvent connotée positivement » (Raveneau 2006b, p. 589).

«Sans dénigrer l'escalade, parce que j'en fais aussi beaucoup, surtout aujourd'hui, quand je partais en terrain d'aventure ou quand j'entreprends une ascension je n'avais pas le sentiment de faire la même chose [...] pour le coup ce n'est pas du tout pareil, l'engagement est plus du tout le même » (Michaël, alpiniste).

Le risque n'a pour autant de sens que s'il est mis en scène, comme dans les activités circassiennes (Fourmaux, 2006). Ainsi, le risque est perçu comme moyen de connaissance et d'expression de soi dans un cadre collectif.

Si l'engagement corporel est un vecteur d'unification existentielle essentiellement individuel, il est donc important de noter que cette construction identitaire ne se produit pour autant dans la solitude puisque, en tant qu'individu revendiquant la sécurité et la responsabilité, nombre de pratiquants s'engagent sous le regard bienveillant du groupe. Il ne saurait alors être question d'envisager l'engagement identitaire comme un processus autonome et totalement individuel.

\subsubsection{Une certaine démesure}

Bien qu'il témoigne rarement d'une inconséquence, et soit au contraire synonyme de l'exercice d'un contrôle, de responsabilité, de grande rigueur sur le plan sécuritaire et de prudence (Martha \& Griffet, 2006; Raveneau, 2006b), l'engagement corporel suppose la captation de mobiles et de motifs d'action puissants - l'envie d'agir - qui interrogent. Même si les pratiquants trop peu prudents sont généralement marginalisés, diverses formes de mise en suspens de l'intégrité corporelle dépassent dans bien des cas ce qui est considéré comme raisonnable aux yeux $\mathrm{du}$ plus grand nombre, allant à l'encontre de ce que la norme sociale tend à imposer. Pourtant, cela ne se traduit pas par un rejet pur et simple, car la démesure, tant qu'elle concerne l'échelle de l'individu, exerce une certaine séduction (Comte-Sponville, 2001) : elle fascine et inspire, là où la mesure suscite généralement l'ennui. L'indéniable succès médiatique des sports dits « extrêmes » en atteste, là où tant d'autres expositions au risque sont désignées comme particulièrement insupportables, inacceptables, déviantes et/ou irresponsables. Une seule et même modalité d'engagement corporel peut ainsi être vue de manière contrastée : preuve de courage et d'audace pour les uns, elle est pour les autres synonyme de transgression déraisonnable des limites assignées à l'Homme.

En tout état de cause, l'engagement corporel revêt aussi une signification morale et idéologique, revendiquée 
par bien des adeptes de sports dangereux : il constitue l'occasion d'affirmer une cohérence avec des valeurs de transgression, de valoriser la liberté comme la responsabilité individuelles, et de dénoncer la dérive des sociétés occidentales vers l'aseptisation et l'hyper-sécuritaire.

Ce type de discours nous renvoie à ce que Raveneau (2006b, p. 582) nomme « l'affirmation viscérale d'une libre disposition de soi assumée jusqu'en ses dernières conséquences ». À travers le risque pris se mesure la valeur attachée à ce pour quoi il a été accepté, et ce qu'on est prêt à risquer pour elle : la patrie dans la guerre, la liberté dans la résistance, l'amour dans le sacrifice de son confort personnel (Ewald, 1999)... Et son intégrité corporelle ou même sa vie dans certaines pratiques sportives.

«Il faut choisir, et choisir c'est...je dirais que c'est accepter ce à quoi on est prêt à renoncer. Par exemple, vous allez choisir une compagne, bon, moi je vous dis que vous n'avez pas choisi tant que vous, vous n'avez pas renoncé. Si vous n'avez renoncé à rien vous n'avez pas choisi. Vous avez renoncé à quoi? Vous avez renoncé à toutes les femmes à qui vous allez plaire, vous comprenez? [...] acceptez ça et vous serez en mesure d'accepter tout. Dans notre pratique c'est finalement un peu pareil. À quoi sommes-nous prêts à renoncer pour cette activité? Jusqu'où sommes-nous prêts à aller? » (Christian, spéléologue).

Morin (1970) évoque ainsi la noblesse de celui qui réussit à contrôler la mort, tout en s'enivrant de la vie et en en jouissant pleinement. Le prix à payer peut être sa propre vie, mais la vie dangereuse comme morale existe bel et bien d'après Morin, en réaction aux excès pacificateurs et prophylactiques qui caractérisent notre quotidien hyper-civilisé. On peut donc aussi s'engager dans une pratique sportive «à risque » dans le but d'être en conformité avec ses valeurs (Corneloup, 1993). Dans ce sens, choisir une activité physique dite à risque constitue pour l'individu-citoyen un moyen pour communiquer physiquement à autrui son goût pour les valeurs libertaires et d'aventure. Ainsi, à travers un style sportif « exposé » ou « engagé », et les références symboliques sur lesquelles il se construit, se joue aussi un positionnement social et axiologique à plus grande échelle, au sein d'un champ d'opinions plurielles. Certains grimpeurs traditionnels « laissent la nature dicter leur route », plutôt que d'utiliser des moyens rationalisés et artificiels d'ascension. Ils maintiennent ainsi sciemment des niveaux élevés de risque et d'incertitude.

\subsubsection{De l'engagement au lifestyle}

Dans certains cas les pratiquants qualifient leur engagement de lifestyle davantage que de sport. Une conception singulière de la pratique, ainsi qu'un style de vie alternatif et distinctif, tout comme un code de communication spécifique donnent une identité particulière et exclusive aux membres de ces communautés. Pour conserver le statut ordinairement conféré aux navigateurs ou aux alpinistes par exemple - basé sur les images de l'aventurier - il convient d'appartenir au cercle privilégié des «vrais », des insiders (Donnelly \& Young, 1988). Car si autrefois réaliser l'ascension du Mont-blanc, une transatlantique, ou emprunter un couloir enneigé hors des pistes était considéré comme un véritable et authentique exploit, aujourd'hui cela ne suffit plus.

L'adhésion à un lifestyle sport va au-delà du simple engouement pour une activité de loisir, et tourne parfois à l'idée fixe.

« Mais c'est vrai qu'arrivé un moment je pense plus qu'à ça selon la saison »(Bruno, apnéiste).

«Perso pendant des années ma vie s'est organisée autour de ma pratique de la montagne. Je bossais en saison à l'endroit ou mes envies me menaient!» (Michaël, alpiniste).

«L'avantage c'est que j'ai des horaires décalés. Tu vois là je travaille en nuit ce qui fait que j'avais toute ma journée de libre. Et puis aussi j'ai des repos décalés. C'est-à-dire qu'on n'a pas tout le temps repos le samedi et le dimanche, c'est souvent des repos en semaine, et comme on fait ces semaines de 6 jours on a des repos de 3 jours. Et ça pour partir à la montagne c'est génial. Donc voilà c'est le seul intérêt» (Hervé, BASE-jumper).

Selon Wheaton (2004), l'agrégation des efforts consentis (temps, argent) et des contraintes acceptées (professionnelles, familiales, sentimentales) constitue un révélateur pertinent du degré d'engagement. Déchargés de toute responsabilité familiale, certains pratiquants, à l'image des kayakistes de haute rivière observés par Marsac (2006) consacrent l'essentiel de leur temps de loisir à cette activité, conformément au schéma plus général rapporté par Lyng (1990). L'engagement suppose ainsi d'être volontaire, aux deux sens du terme, puisqu'il faut de la constance, de la persévérance, et parfois de l'opiniâtreté pour le construire et le soutenir dans la durée. Savoir faire face à des situations incertaines implique par exemple un long apprentissage technique, ainsi que l'expérimentation préalable de situations limite, voire d'incidents et/ou accidents.

\section{Conclusion}

Parler d'engagement corporel revêt a minima un double sens : l'idée d'un jeu et d'une mise en jeu acceptée de l'intégrité corporelle (dimension pragmatique), doublée du cheminement plus personnel induit par la gageure (dimension identitaire). Dans une telle perspective, s'interroger sur l'engagement corporel revient à questionner le lien qui unit des conduites dangereuses aux représentations que leurs adeptes en ont, c'est-à-dire à s'interroger sur 
la relation entre la construction de l'acte d'engagement et celle de l'identité de celui qui se vit à travers lui. En reprenant Ewald (1999), il semble que c'est la possibilité, pour les pratiquants engagés, de prendre des risques qui leur permet de ne pas se résumer à leur stricte existence biologique. Par conséquent, la confrontation aux dangers, librement consentie, se pose en tant que support d'action et de construction identitaire.

\section{Bibliographie}

Bajoit, G. (2003). Le changement social. Approche sociologique des sociétés occidentales contemporaines. Paris : Armand Colin.

Barthélemy, M. (2002). Les aventures organisées ou l'illusion $\mathrm{du}$ risque. L'exemple du Marathon des sables. Cahiers Espaces, 73, 61-67.

Berthelot, J.-M. (2002). Pour un programme sociologique non réductionniste en étude des sciences. Revue Européenne des Sciences Sociales, XL(124), 233-252.

Boudon, R. (1990). L'art de se persuader des idées fausses, fragiles ou douteuses. Paris : Fayard.

Bouet, M. (1968). La signification du sport. Paris : Éditions Universitaires.

Bourdin, A. (2003). La modernité du risque. Cahiers Internationaux de Sociologie, 1(114), 5-26.

Boutroy, E. (2002). Une technique du vertige? Les usages du corps dans une pratique ascensionniste : la via ferrata. Techniques et Cultures, 39, 121-138.

Boutroy, E. (2006). Cultiver le danger dans l'alpinisme himalayen. Ethnologie Française, XXXVI(4), 591-601.

Coakley, J. (2004). Sports in Society: Issues and controversies. New York : MacGraw-Hill.

Coanus, T., \& Pérouse, J.-F. (1999). Villes et risques. Regards croisés sur quelques cités « en danger». Paris : Economica/Anthropos.

Collard, L. (1997). Approche sociologique des sports à risque. STAPS, 18(44), 83-96.

Collard, L. (1998). Sports, enjeux et accidents. Paris : P.U.F.

Comte-Sponville, A. (2001). Dictionnaire philosophique. Paris : P.U.F.

Corneloup, J. (1993). Les nouvelles formes de sociabilité en escalade, Actes du colloque Sport, relations sociales et action collective (pp. 199-210). Talence : MSHA.

De Léséleuc, E., \& Raufast, X. (2004). Du risque en sports de nature : une approche sociopsychanalytique. Revue Européenne de Management du Sport, 12, 237-258.

Donnelly, P., \& Young, K. (1988). The construction and confirmation of identity in sport subcultures. Sociology of Sport Journal, 5, 223-240.

Douglas, M. (1992), Risk and danger Risk and blame : Essays in cultural theory (pp. 38-54). London : Routledge.
Duchastel, J., \& Laberge, D. (1999). La recherche comme espace de médiation interdisciplinaire. Sociologie 85 Sociétés (31), 63-76.

Duclos, D. (2005). Analyse critique du champ socio-politique des risques techniques et sociaux. Disponible à http:// www.geo-anthropology.com/attachment/155695

Ewald, F. (1999). Les fondements moraux de la valeur du risque. In C. Carde et al. (Eds.), Risque et Société. Gif-sur-Yvette, France : Nucléon.

Ewald, F., \& Kessler, D. (2000). Les noces du risque et de la politique. Le Débat, 109, 55-72.

Fourmaux, F. (2006). Le nouveau cirque ou l'esthétisation du frisson. Ethnologie Française, XXXVI(4), 659-668.

Giddens, A. (1987). La constitution de la société : éléments de la théorie de la structuration. Paris : P.U.F.

Giddens, A. (1991). Modernity and self-identity: Self and society in the labe modern age. Cambridge: Polity Press.

Gilbert, C. (2003). La fabrique de risques. Cahiers Internationaux de Sociologie, CXIV, 55-72.

Griffet, J. (1991). La sensibilité aux limites. Paris : Société.

Héas, S., Lebreton, F., Frezza, S., Bodin, D., \& Robène, L. (2007). Des sports extrêmes aux professions risquées. Revue des Sciences Sociales(38).

Jardine, C. G., \& Hrudey, S. E. (1997). Mixed messages in risk communication. Risk Analysis, 17(4), 489-498.

Kaplan, S. (1997). The words of risk analysis. Risk Analysis, $17(4), 407-417$.

Kaplan, S., \& Garrick, J., B. (1981). On the quantitative definition of risk. Risk Analysis, 1, 1.

Kay, J., \& Laberge, S. (2003). Oh say can you ski? Imperialistic construction of freedom in Warren Miller's Freeriders. In R. Rinehart \& S. Sydnor (Eds.), To the extreme. Alternative Sports, inside and out (pp. 381-398). Albany : State University of New York Press.

Ladrière, J. (1997). L'éthique dans l'univers de la rationalité. Namur : Artel / Fides.

Le Breton, D. (2002). Conduites à risque. Paris : PUF.

Leiss, W., \& Chociolko, C. (1994). Risk and responsability. Montréal, \& Kingston : McGill Queen's University Press.

Liardet, A., \& Raveneau, G. (2006). Toujours ramener le bateau au port. Ethnologie Française, XXXVI(4), 677-680.

Lyng, S. (1990). Edgwork: A social psychological analysis of volontary risk taking. American Journal of Sociology, 95(4), 851-866.

Marsac, A. (2006). En kayak de haute rivière : pratiques individuelles et engagements partagés. Ethnologie Française, $X X X V I(4), 603-611$.

Martha, C., \& Griffet, J. (2006). Sauter dans le vide : le Base-Jump, le jeu le plus sérieux du monde. Ethnologie Française, XXXVI(4), 635-642.

Monod, H., \& Kahn, J.-F. (2005). Médecine du sport. Paris : Masson.

Morin, E. (1970). Journal de Californie. Paris : Seuil. 
Needham, R. (1975). Polythetic classification: convergence and consequences. Man (10), 349-369.

Olivier de Sardan, J.-P. (1998). Emique. L'Homme, 38(147), 151-166.

Pardo, M. (2002). Sociologie et risque : nouveaux éclairage sur les facteurs sociaux et la participation publique. MANA, Revue de Sociologie et d'Anthropologie (10-11), 285-305.

Parlebas, P. (1981). Contribution à un lexique commenté en sciences de l'action motrice. Paris : INSEP.

Passeron, J.-C. (2006). Le raisonnement sociologique. Un espace non poppérien de l'argumentation. Paris : Albin Michel.

Penin, N. (2004). Prises de risque sportif : saisir des modes d'engagement sexués. Colloque Acteur, risque et prise de risque à l'épreuve des sciences sociales, CLERSE-IFRESI, Lille, France.

Poizat, D. (2000). Hors-piste : comportements, connaissances et perceptions des pratiquants. Neige $\&$ Avalancges, 89, $2-8$.

Raveneau, G. (2006a). La plongée sous marine, entre neutralisation du risque et affirmation de la sécurité. Ethnologie Française, XXXVI(4), 613-623.

Raveneau, G. (2006b). Prises de risque sportives : représentations et constructions sociales. Ethnologie Française, XXXVI(4), 581-586.

Renn, O. (1998). Three decades of risk reseach, accomplishments and new challenges. Journal of Risk Research, 1, 49-71.

Rotillon, G. (2002). L'alpinisme et l'escalade dans l'économie des loisirs. In M. Baddeley (Ed.), Sports extrêmes, sportifs de l'extrême. La quête des limites (pp. 141-157). Genève : Georg éditeur.
Routier, G. (2011). De l'engagement au désengagement corporel. Une approche sociologique plurielle des dynamiques, ruptures et permanences identitaires face à l'acceptation du danger dans les sports de nature. Thèse de doctorat, Université Européenne de Bretagne-Rennes 2, France. Disponible à http://tel.archives-ouvertes.fr/tel-00628608

Seigneur, V. (2004). L'acteur preneur de risque: des figures comportementales multiples? Les problèmes de définition $d u$ risque. Colloque Acteur, risque et prise de risque à l'épreuve des sciences sociales, CLERSE-IFRESI, Lille, France.

Soulé, B. (2008). Les « sports extrêmes » : analyse terminologique d'une caractérisation sportive à succès. Sciences et Motricité, 1 (63), 83-90.

Soulé, B., \& Corneloup, J. (2007a). La conceptualisation en sociologie : influences paradigmatiques et implications méthodologiques. L'exemple de la notion de risque dans le sport. Bulletin de Méthodologie Sociologique (93), 28-54.

Soulé, B., \& Corneloup, J. (2007b). Sociologie de l'engagement corporel. Risques sportifs et pratiques « extrêmes» dans la société contemporaine. Paris : Armand Colin.

Stranger, M. (1999). The aesthetics of risk. A study of surfing. International Review for the Sociology of Sport, 34(3), 265-276.

Wheaton, B. (2004). Understanding lifestyle sports: Consumption, identity and difference. London : Routledge.

Weber, M. (1995). Économie et Société. Paris : Pocket.

Young, K., \& Atkinson, M. (2008). Tribal Play. Sports subcultures (Vol. 4). London : Elsevier Press. 